\title{
Reproducción y alimentación de Phytotoma raimondii, cortarrama peruana en El Gramadal, Ancash
}

\author{
Reproduction and food habits and of Phytotoma raimondii, Peruvian \\ Plantcutter in El Gramadal, Ancash
}

1 Asociación Peruana para la
Conservación de la Naturaleza
-APECO, Parque José de Acosta
187, Magdalena, Lima 17, Perú.
Email Mario Rosina:
kraken54@gmail.com
2 Email Mónica Romo:
romomonica@gmail.com

Presentado: 25/01/2012 Aceptado: $\quad 30 / 09 / 2012$

Publicado online: 09/11/2012

\author{
Mario Rosina ${ }^{1}$ y Mónica Romo²
}

\begin{abstract}
Resumen
Se confirma la existencia de un buen hábitat con buena disponibilidad de alimento y de sitios para reproducción de la cortarrama peruana en El Gramadal (Huarmey). Las dos especies más importantes para su alimentación "algarrobo" (Prosopis pallida) y "palo negro" (Grabowskia boerhaviifolia), se encuentran en suficiente cantidad en el lugar. Esta última planta también es crucial para la reproducción, ya que todos los nidos hallados fueron construidos en esta especie. Hasta el momento se han encontrado nueve nidos entre los años 2010 y 2012 entre diciembre y abril: siete nidos activos, 2 el 2010 (abril), 4 el 2011, (marzo y diciembre) y uno el 2012 (enero). También se encontraron dos nidos vacíos el 2011 (marzo y junio), además de volantones e individuos juveniles desde diciembre a junio.
\end{abstract}

Palabras clave: Phytotoma raimondii; Peruvian Plantcutter; Cortarrama peruana; nidos; volantones; Grabowskia boerhaviifolia; palo negro.

\section{Abstract}

The occurrence of good habitat with suitable food and sites for reproduction for the Peruvian plantcutter at El Gramadal (Huarmey) is confirmed. The two most important species for food "algarrobo" (Prosopis pallida) and "palo negro" (Grabowskia boerhaviifolia) both occur in sufficient amount in this place. The last species is also crucial for the reproduction since all the nests found were in this species. To date 9 nests have been found during 2010 and 2012 and between December and April: seven active nests, 2 in 2010 (April) and 4 in 2011(March and December) and 1 in 2012 (January). In addition, two empty nests were found in 2011 (March and June), and fledglings and juveniles from December to June.

Keywords: Phytotoma raimondii; Peruvian Plantcutter; nest; chicks; flendglings; Grabowskia boerhaviifolia; palo negro.

\section{Introducción}

La cortarrama peruana Phytotoma raimondii Taczanowski 1883, es un ave endémica y de distribución restringida a la costa norte del Perú, desde Piura hasta Ancash, hasta los $300 \mathrm{~m}$ de altitud (Schulenberg et al. 2010) aunque otros autores mencionan que llega hasta los $550 \mathrm{~m}$ (Clements \& Shany 2001). Esta especie está en peligro de extinción debido sobre todo a la pérdida de su hábitat. El lugar más al sur donde se le ha encontrado en la actualidad es el sitio conocido como El Gramadal en la provincia de Huarmey, departamento de Ancash, donde la población sería de aproximadamente 80 individuos.

Se conoce de sus hábitos alimenticios y reproducción por observaciones realizadas en los departamentos de Piura, Lambayeque, La Libertad y ahora en Ancash. En un trabajo sobre su dieta en Talara (Abramonte 2007) se indica que la cortarrama peruana se alimenta de 10 especies de plantas, las que fueron encontradas por observación directa, lavado del tubo digestivo o en heces. En el Santuario Histórico Bosque de Pomac se menciona que se alimenta de "canutillo" o "palo negro" (Grabowskia boerhaaviifolia; E. Sanchez com. pers). En este trabajo reportaremos la dieta observada en El Gramadal y también las similitudes halladas con las observaciones en los sitios anteriormente mencionados.

Sobre la reproducción, Abramonte (2007) cita que ésta ocurre entre enero y marzo en Talara y describe la conducta de cortejo y cópula así como el proceso de construcción del nido por ambos padres. En el Gramadal ya se han descrito dos nidos hallados el
2010 (Rosina \& Romo 2010), los primeros reportados desde 1934 (Flanagan \& Millen 2008).

El objetivo de este trabajo es ampliar el conocimiento de la historia natural de la cortarrama peruana, específicamente de la alimentación y reproducción en el sitio El Gramadal.

\section{Material y métodos}

Lugar de estudio.- El trabajo fue realizado en El Gramadal, distrito de Huarmey, provincia de Huarmey, Departamento de Ancash. El sitio es aparentemente el límite sur de la distribución de esta ave y no está registrado en la más reciente publicación sobre la distribución del Phytotoma raimondii (Flanagan et al. 2009).

La mayor densidad poblacional del ave y la mejor zona de anidación se encuentra en la porción Este, llamada desde ahora El Gramadal 1, siendo la del lado Oeste El Gramadal 2. Hemos estimado una población de 40 individuos en El Gramadal 1 y otros 40 individuos en El Gramadal 2 (Fig. 1).

La superficie de ambos sitios es de aproximadamente 134 ha, teniendo la zona central o núcleo de El Gramadal 1, 17 ha, y El Gramadal 2, una extensión de 127 ha respectivamente. Mientras El Gramadal 1 tiene un suelo formado por sedimento aluvial proveniente de un antiguo cauce de avenida, hoy seco, El Gramadal 2 tiene un suelo donde predomina la arena acarreada por el viento desde el litoral y abundan las pequeñas dunas, debido a su cercanía de la orilla marina. Este trabajo está centrado principalmente en el sitio que hemos denominado El Gramadal 1 para el caso de las mediciones de nidos. 


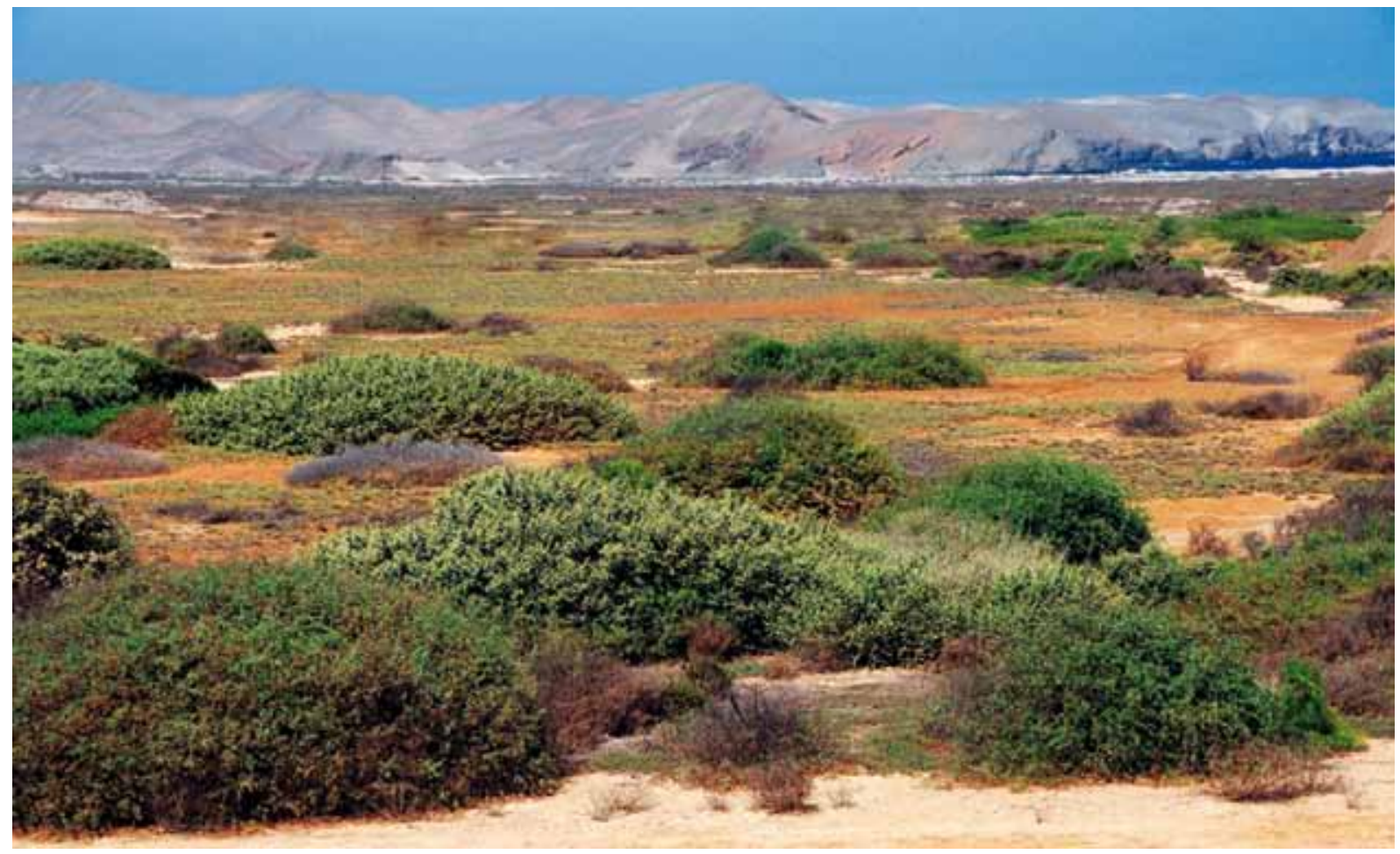

Figura 1. Paisaje de El Gramadal en Ancash (foto M.Tweddle).

El hábitat consta sobre todo de grama salada, arbustos achaparrados y espinos, encontrándose un total de 11 especies vegetales. Además de registrar las especies en el área hicimos dos parcelas de 0,5 hectáreas cada una en El Gramadal 1 y otras dos parcelas de iguales dimensiones en El Gramadal 2 para hallar la densidad de plantas. En cada parcela se contaba el número de individuos de cada especie de arbusto o árbol. No se contó la Distichlis spicata grama salada, especie que aparentemente carece de importancia para la presencia y abundancia del ave (Tabla 1). Además de las plantas del sitio, también anotamos las especies de aves con las que la cortarrama peruana comparte el lugar (Tabla 2).

Se visitó El Gramadal los días 29 de noviembre y 28 de diciembre del 2009, 2 de enero, 1, 6, 13 y 14 de abril, 7 y 10 de

Tabla 1. Especies de flora y número de individuos de arbustos y árboles en las dos parcelas en El Gramadal 1 y el Gramadal 2.

\begin{tabular}{|c|c|c|c|c|c|c|c|c|}
\hline Especie & $\begin{array}{l}\text { Nombre } \\
\text { común }\end{array}$ & $\begin{array}{l}\text { Forma de } \\
\text { vida }\end{array}$ & $\begin{array}{l}\text { Interacción con la } \\
\text { cortarrama }\end{array}$ & $\begin{array}{c}\text { Estado de conservación } \\
\text { de DGFF }\end{array}$ & G1 & G2 & $\mathrm{G}^{2}$ & G2 \\
\hline Grabowskia boerhaviifolia & $\begin{array}{c}\text { palo } \\
\text { negro o } \\
\text { canutillo } \\
\end{array}$ & arbusto & $\begin{array}{l}\text { alimento (hojas } \\
\text { y frutos), nido, } \\
\text { refugio, posadero. }\end{array}$ & & $x$ & $x$ & 21,24 & 14,4 \\
\hline Capparis scabrida & zapote & arbusto & $\begin{array}{c}\text { posadero, } \\
\text { alimento? (hojas) }\end{array}$ & En peligro crítico (CR) & $x$ & & 3,3 & \\
\hline Capparis cordata & satuyo & arbusto & posadero, refugio & & $x$ & $x$ & 5,9 & 5,5 \\
\hline Schinus molle & molle & árbol & $\begin{array}{l}\text { posadero, refugio, } \\
\text { material para nido }\end{array}$ & & $x$ & & 2,2 & \\
\hline Prosopis pallida & algarrobo & $\begin{array}{c}\text { árbol } \\
\text { achaparrado }\end{array}$ & $\begin{array}{l}\text { alimento (flores, } \\
\text { ramitas tiernas y } \\
\text { brotes), posadero, } \\
\text { refugio }\end{array}$ & Vulnerable (VU) & $x$ & $x$ & 0,1 & \\
\hline Acacia macracantha & faique & $\begin{array}{c}\text { árbol } \\
\text { achaparrado }\end{array}$ & posadero & Casi amenazado (NT) & $x$ & & 8,2 & \\
\hline Distichlis spicata & $\begin{array}{l}\text { grama } \\
\text { salada }\end{array}$ & hierba & & & $x$ & $x$ & & \\
\hline Cryptocarpus pyriformis & chope & arbusto & & & $x$ & $x$ & & 0,5 \\
\hline Cordia lutea & overo & arbusto & & & & $x$ & & \\
\hline Galvezia fruticosa & & arbusto & & & $x$ & & & \\
\hline Ficus carica & higo & arbusto & & Introducida & & $x$ & & \\
\hline Total & & & & & 9 & 7 & 6 & 3 \\
\hline
\end{tabular}

1= Dirección General Forestal y de Fauna, Ministerio de Agricultura. 2= número de individuos en parcelas de 0,5 ha. 
Tabla 2. Lista de aves de El Gramadal

\begin{tabular}{|c|c|c|}
\hline Familia & Especies & \\
\hline Cathartidae & 1. & Cathartes aura \\
\hline FALCONIDAE & 2. & Falco sparverius \\
\hline BURHINIDAE & 3. & Burhinus superciliaris \\
\hline Columbidae & 4. & Zenaida auriculata \\
\hline StRIGIDAE & 5. & Athene cunicularia \\
\hline CAPRIMULGidAe & 6. & Chordeiles acutipenis \\
\hline TrochiLIDAE & 7. & Rhodopis vesper \\
\hline Trochilidae & 8. & Amazilia amazilia \\
\hline TROCHILIDAE & 9. & Myrtis fanny \\
\hline TROCHILIDAE & 10. & Thaumastura cora \\
\hline FURNARIDAE & 11. & Geositta peruviana \\
\hline TyRANNIDAE & 12. & Pyrocephalus rubinus \\
\hline TYRANNIDAE & 13. & Muscigralla brevicauda \\
\hline TYRANNIDAE & 14. & Tyranus melancholicus \\
\hline РнутотоміDAe & 15. & Phytotoma raimondii \\
\hline HIRUNDINIDAE & 16. & Pygochelidon cyanoleuca \\
\hline Mimidae & 17. & Mimus longicaudatus \\
\hline Coerebidae & 18. & Coereba flaveola \\
\hline Thraupidae & 19. & Conirostrum cinereum \\
\hline
\end{tabular}

octubre del 2010, 7, 8 y 27 de marzo, 16, 17 y 18 de junio, 23 de julio, 1 de agosto, 26 de diciembre del 2011 y 16 de enero del 2012 totalizando 19 días en 15 visitas.

Alimentación.- La cortarrama peruana es un ave exclusivamente vegetariana que utiliza su pico de bordes aserrados para cortar las ramitas, brotes, hojas y frutos de los que se alimenta. Cada vez que se visitó el lugar se buscó indicios de alimentación en las plantas y se observó las que consumía. Para el caso del algarrobo, se registró la posición geográfica de esta especie vegetal mediante GPS.

Reproducción.- En cada visita a El Gramadal se buscó exhaustivamente nidos en los arbustos y/o conductas de la hembra o el macho que indicaran construcción del nido o incubación, así mismo se observó si había presencia de volantones o individuos juveniles.

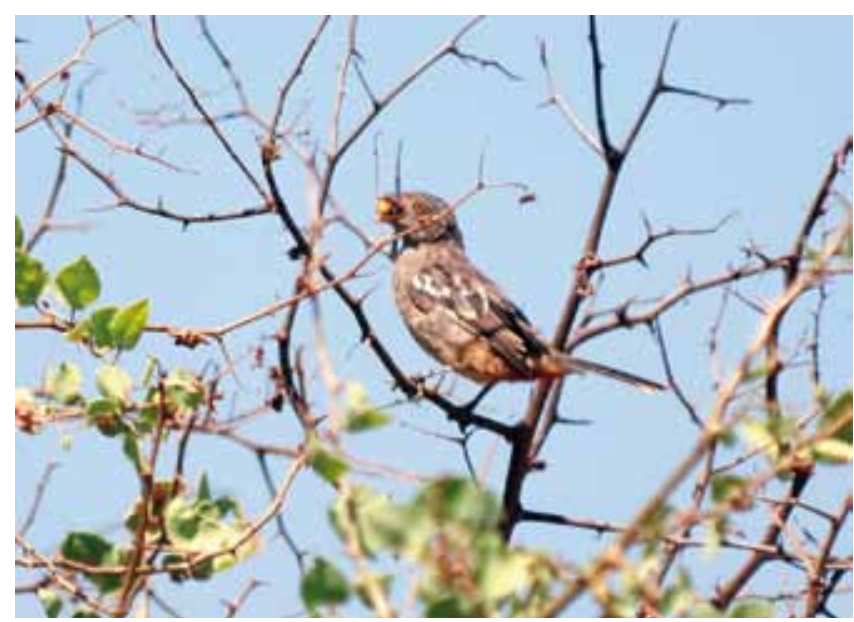

Figura 2. Phytotoma raimondii, Cortarrama peruana comiendo fruto de palo negro(foto M.Tweddle).

\section{Resultados}

Hábitat.- De las 11 especies vegetales presentes en el área, la cortarrama peruana tiene alguna interacción al menos con 6 de ellas ya sea como alimento, lugar de nidificación, posadero, refugio o material para construcción del nido. En la Tabla 1 se muestran las especies de arbustos y árboles del lugar y el número de individuos encontrados en cada una de las 2 parcelas de 0,5 ha y su relación con el ave. En algunos casos algunas especies presentes se encontraban fuera de las parcelas. El algarrobo (Prosopis pallida, Fabaceae), presente en este sitio con individuos achaparrados y en forma arbustiva, se encuentra en una de las dos parcelas con 1 individuo, pero en las 4 ha donde está el núcleo de El Gramadal 1 hay 4 individuos. En El Gramadal 2 también se encuentran 8 individuos en una superficie de 4 ha. La segunda especie vegetal de gran importancia para el ave es el palo negro o canutillo (Grabowskia boerhaaviifolia, Solanacea), que es el arbusto predominante en el lugar, mide hasta $3 \mathrm{~m}$ de altura, es espinoso y muy enmarañado y es muy importante para la reproducción de la cortarrama peruana, ya que en esta planta se encontraron los 9 nidos. La densidad del "palo negro" en dos parcelas de media hectárea es de 21 y 24 individuos respectivamente en El Gramadal 1 y de 4 y 14 individuos en El Gramadal 2. Otras especies presentes de importancia son Capparis cordata "satuyo", Capparis scabrida "zapote", Schinus molle "molle" y Acacia macracantha "huarango".

Alimentación.- La dieta de la cortarrama peruana parece variar de acuerdo a la disponibilidad estacional de las plantas de su hábitat. En El Gramadal, hemos observado que el algarrobo tiene brotes y se observan ramitas comidas desde mayo a enero y sería uno de los alimentos más importantes durante gran parte del año tanto para los adultos como para los juveniles. En noviembre hemos observado además flores de algarrobo comidas. Por otro lado hemos visto al ave alimentándose de los frutos y hojas del palo negro (Fig. 2 y 3). Esta planta, se encontraba fructificando cada vez que visitamos el lugar y siempre hubo alguna con mayor abundancia de frutos muy maduros, sobre todo en los meses más calurosos del año (noviembre a junio). En diciembre y enero encontramos heces con semillas de palo negro. Los juveniles también se alimentan de brotes, hojas y ramitas tiernas de algarrobo, además de hojas y frutos maduros de palo negro. La densidad de ambas plantas es alta en El Gramadal 1y la distancia entre éstas en la zona de estudio es corta, lo que facilita el vuelo de los volantones de un arbusto a otro.

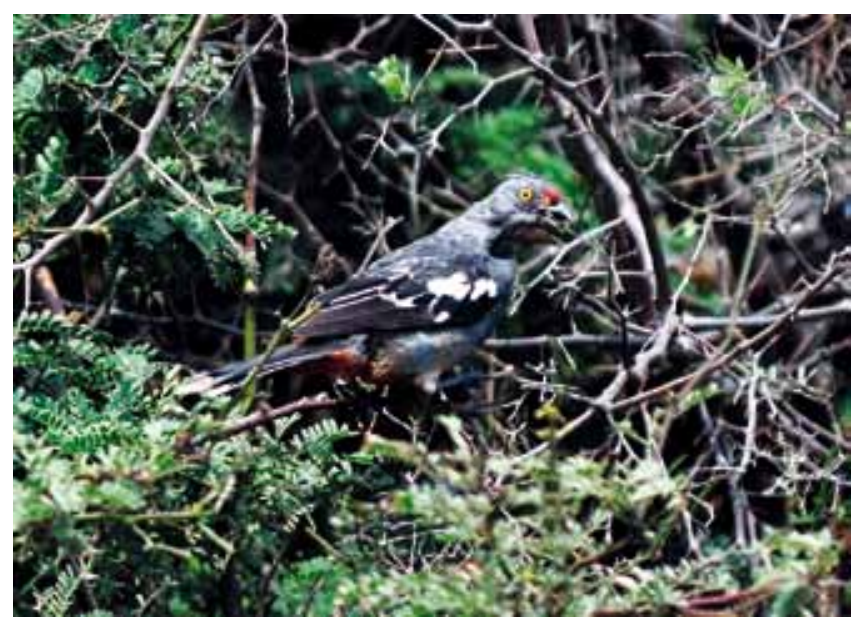

Figura 3. Phytotoma raimondii, Cortarrama peruana comiendo hoja de palo negro(foto M.Tweddle). 


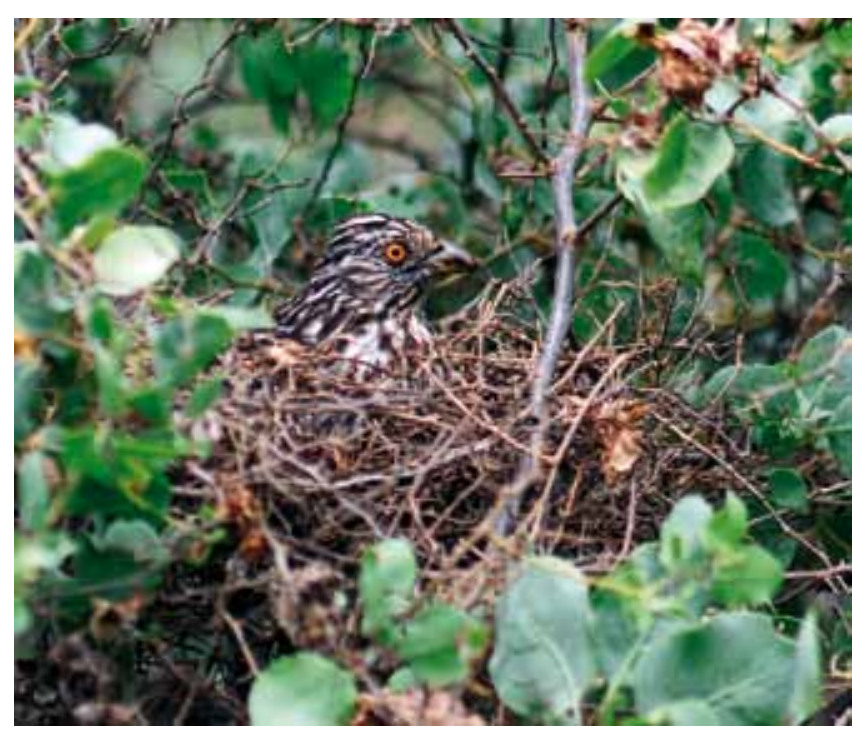

Figura 4. Phytotoma raimondii, nido 4 , hembra anidando en palo negro (foto M.Tweddle)

Nidos.- La construcción del nido y la alimentación de los pichones están a cargo de ambos padres, mientras que la incubación corresponde exclusivamente a la hembra (Fig. 4). La madre suele ausentarse del nido aun con los pichones recién nacidos a veces hasta por 20 minutos. El macho suele percharse en una rama alta cercana al nido en actitud vigilante, vocalizando constantemente y no permite la presencia de otras cortarramas en su territorio, siendo este comportamiento muy marcado durante la reproducción. Cuando no se están reproduciendo los machos se exhiben menos, así mismo sus reclamos disminuyen en frecuencia e intensidad. No hemos podido observar apareamientos.

Los nidos en forma de plato son amplios y poco profundos, miden unos $15 \mathrm{~cm}$ de diámetro y su estructura y diseño facilita el pronto abandono de éste por parte de los pichones. Todos los nidos encontrados estaban construidos sobre palo negro. Son fabricados con palitos y ramitas secas de palo negro en la estructura, molle y algarrobo en la superficie o cama y se disimulan perfectamente con las ramas del arbusto palo negro. Los nueve nidos hallados estaban a una altura promedio de 1,52 $\pm 0,30 \mathrm{~m}$ y un rango entre $1,20-2,20 \mathrm{~m}$ respectivamente, estando ocho de ellos entre 1,20 y 1,70 m de altura, el nido 7 encontrado el 16 de junio de 2011 estaba a 2,20 m del suelo. Estas medidas son también un reflejo de la altura de los arbustos

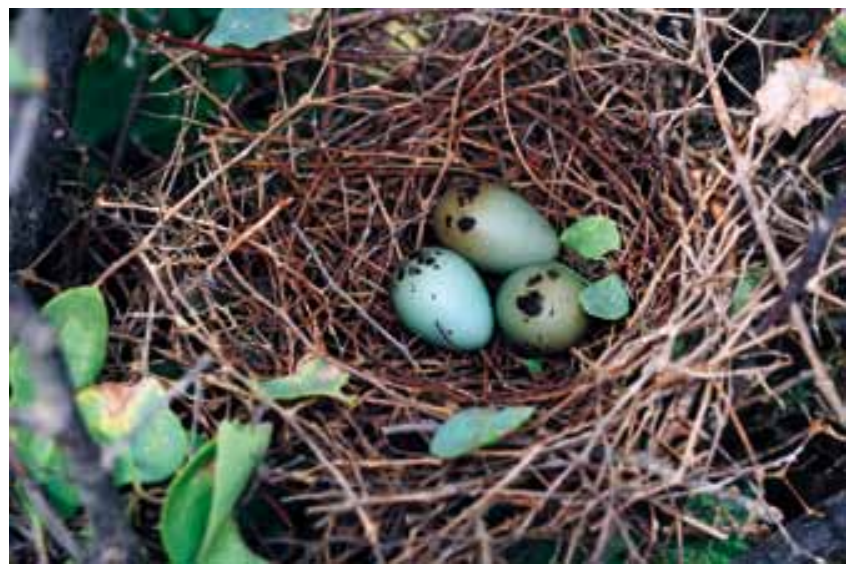

Figura 5. Phytotoma raimondii, Nido 5 el 8 de marzo de 2011 (foto M. Tweddle). de palo negro del lugar. En la Tabla 3 se lista la altura de los nidos, número de huevos, pichones y volantones encontrados en el 2010, 2011 y 2012.

La hembra pone de 2 a 3 huevos de color celeste claro con manchas marrones en el polo romo (Fig. 5). Según nuestras observaciones la incubación podría durar entre 13 y 19 días. La descripción de los primeros huevos y volantones en este lugar de estudio se encuentra en Rosina y Romo (2010); luego de estos, hemos encontrado en el 2011 otros seis nidos más (nidos 3 al 8) y uno el 2012 (nido 9) con similares características. Dos de ellos ya estaban inactivos cuando fueron encontrados (27 marzo, nido 6 y 16 de junio, nido 7). En diciembre del 2011 se encontró un nido con un pichón emplumando y dos huevos de color beige, que podrían ya estar perdidos debido a la diferencia que tendrían si eclosionan con el pichón nacido varios días antes, el mismo que al momento de ser encontrado tendría aproximadamente de siete a diez días de edad (Fig. 6). Volvimos a visitar el nido el 16 de enero de 2012 y en efecto el pichón ya había abandonado el nido y podía observársele como volantón en un arbusto cercano, mientras que los 2 huevos, decolorados por el sol aún estaban en el nido. Hemos observado volantones de los nidos 2, 3 y 4 entre marzo y abril del 2011, además en tres oportunidades observamos otros volantones sin haber encontrado el nido correspondiente (El Gramadal 2: 17 de junio del 2011, 2 volantones; El Gramadal 1: 26 de diciembre del 2011, 3 volantones y 2 de enero del 2012, 2 volantones). Se calcula que de los dos nidos encontrados el 2010 se habrían logrado 3 volantones (75\% de los huevos). De los nidos 3, 4, 5 y 8 del 2011, que contenían un total de 10 huevos, 6 (60\%) lograron convertirse en volantones. Estos 9 volantones más los 5 observados sin haber encontrado el nido significaría que al menos 14 juveniles se produjeron hasta el momento (de abril del 2010 a enero del 2012) (Fig. 7).

Los pichones, nidícolas, son de color negro y empiezan a abandonar el nido antes de los 20 días de nacidos, aunque no dejan el arbusto en el que nacieron, moviéndose y ocultándose dentro de él (Fig. 8). El palo negro por su estructura enmarańada y espinosa les brinda una óptima protección contra los depredadores. Poco después, ya volantones, siguen a los padres por los arbustos cercanos al del nido reclamándoles alimento y también intentando alimentarse por sí mismos de hojas y frutos de palo negro, estos últimos parecerían todavía muy duros y difíciles de arrancar de su pedúnculo cuando no están maduros. La distancia entre los dos

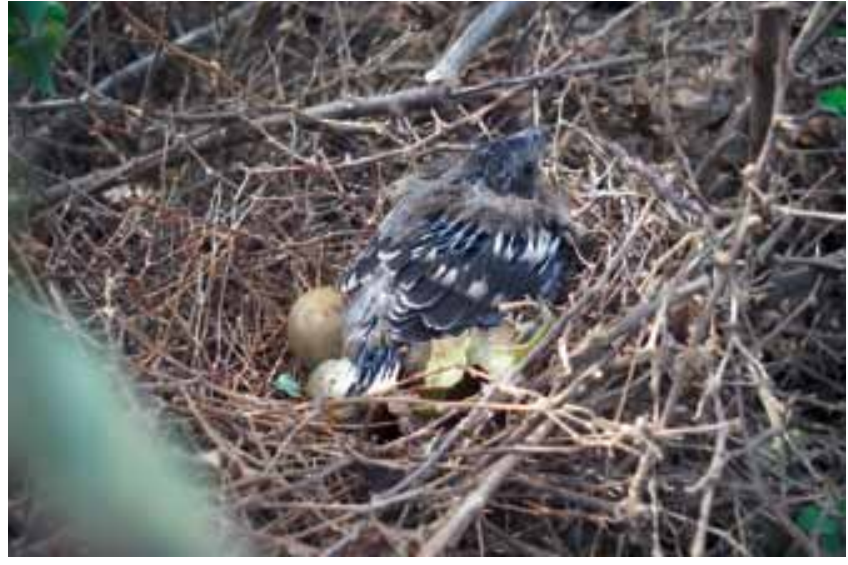

Figura 6. Phytotoma raimondii, Nido 8 el 26 de diciembre de 2011 (foto M. Rosina). 


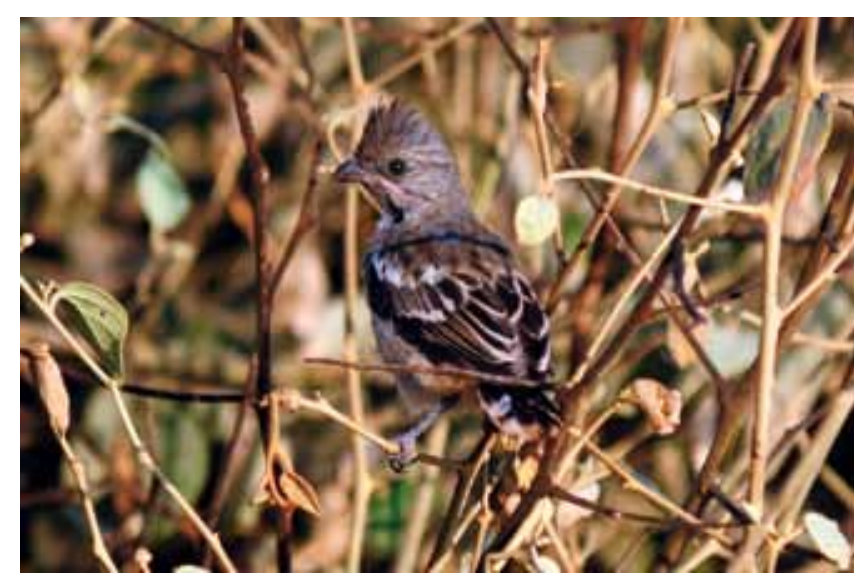

Figura 7. Volantón de Phytotoma raimondii, cortarrama peruana (foto M.Tweddle).

nidos encontrados el 2010 es de 271 m y la distancia más corta y más larga entre los nidos encontrados los primeros meses del 2011, (nidos 3 al 7) es de 21 a 151 m respectivamente, siendo el promedio de $92,7 \pm 42,3 \mathrm{~m}$. Es decir la distancia entre los límites de cada territorio podría ser de 20 a $150 \mathrm{~m}$.

Siendo el algarrobo una especie importante como alimento durante los meses siguientes a la salida de los pichones del nido, se calculó el promedio de distancia entre los nidos y los algarrobos más cercanos. El promedio de la distancia de los 9 nidos al algarrobo más cercano es de $45 \mathrm{~m}$ y el promedio de distancia de los nidos a los dos algarrobos más cercanos es de $52 \mathrm{~m}$. El nido

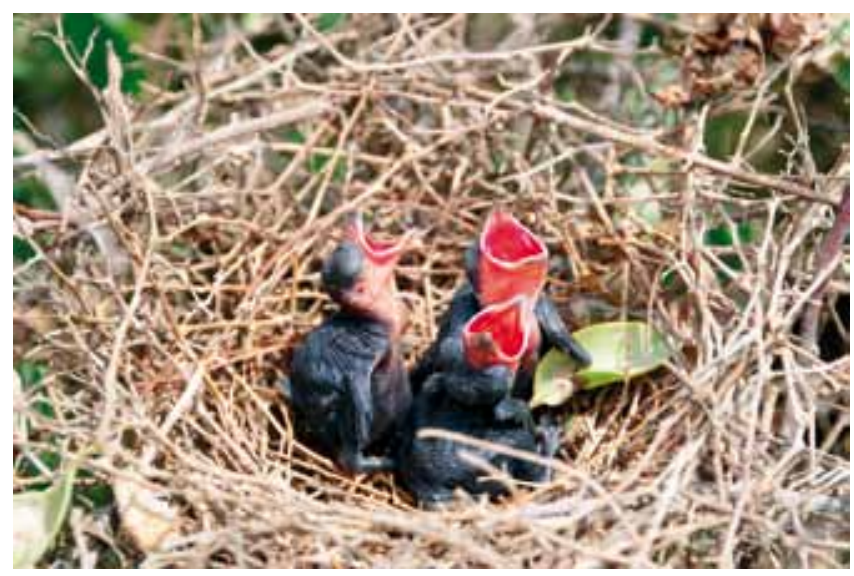

Figura 8. Phytotoma raimondii, Nido 5 el 27 de marzo de 2011(foto M. Tweddle).

9 hallado el 16 de enero del 2012 se encontraba en el mismo arbusto donde estuvo el nido 3 en marzo del 2011, solo que fue construido mas al interior del arbusto, aunque casi a la misma altura (1,60 m y 1,70 $\mathrm{m}$ respectivamente).

Durante la época reproductiva se observa que el plumaje de las hembras y los machos es más oscuro, sobre todo las listas marrones de éstas. Así mismo, el color gris y las marcas de color rufo de los machos son más intensos. Es posible que antes de la reproducción los adultos muden su plumaje y conforme transcurre el año éste se vaya decolorando por la exposición a la luz solar y el desgaste natural de las plumas.

Tabla 3. Características de los 9 nidos de Phytotoma raimondii encontrados en El Gramadal el 2010, 2011 y 2012.

\begin{tabular}{|c|c|c|c|c|c|c|c|}
\hline Nido & Fecha & $\begin{array}{c}\text { Altura } \\
\text { nido (m) }\end{array}$ & $\begin{array}{c}\mathrm{N}^{\circ} \\
\text { huevos }\end{array}$ & $\begin{array}{l}\mathrm{N} .^{\circ} \\
\text { pichones }\end{array}$ & $\begin{array}{l}\mathrm{N} .^{\circ} \\
\text { volantones }\end{array}$ & $\begin{array}{l}\text { Fue depredado } \\
\text { o fracaso }\end{array}$ & Observaciones \\
\hline 1 & $01 / 04 / 10$ & 1,20 & 1 & & & & \\
\hline 1 & $07 / 04 / 10$ & 1,20 & 2 & & & & \\
\hline 1 & $13 / 04 / 10$ & 1,20 & & & & 1 & Se encontró un huevo eclosionado en el nido \\
\hline 2 & $07 / 04 / 10$ & 1,20 & & 2 & & & 2 pichones de 10 a 15 días de nacidos. \\
\hline 2 & $14 / 04 / 10$ & 1,20 & & & 2 & 0 & \\
\hline 3 & $07 / 03 / 11$ & 1,60 & 2 & & & & \\
\hline 3 & $27 / 03 / 11$ & 1,60 & & & 1 & 1 & \\
\hline 4 & $07 / 05 / 11$ & 1,65 & 2 & & & & \\
\hline 4 & $27 / 03 / 11$ & 1,65 & & & 1 & 1 & $\begin{array}{l}\text { Se colectó } 1 \text { huevo perforado, } 1 \text { huevo } \\
\text { eclosionó. }\end{array}$ \\
\hline 5 & 08/03/11 & 1,30 & 3 & & & & \\
\hline 5 & $27 / 03 / 11$ & 1,30 & & 3 & & & 3 pichones de 1 a 2 días de nacidos. \\
\hline 6 & $27 / 03 / 11$ & 1,20 & $i$ & & & $i$ & Nido vacío \\
\hline 7 & $16 / 06 / 11$ & 2,20 & $i$ & & & $i$ & Nido vacío \\
\hline 8 & $26 / 12 / 11$ & 1,65 & 2 & 1 & & 2 & $\begin{array}{l}2 \text { huevos no eclosionaron y } 1 \text { pichón de } 7 \text { a } 10 \\
\text { días de nacidos. }\end{array}$ \\
\hline 8 & $16 / 01 / 12$ & & & & 1 & 1 & Volantón en el arbusto. \\
\hline 9 & $16 / 01 / 12$ & 1,70 & 2 & & & & En el mismo arbusto que nido 3 \\
\hline
\end{tabular}


Tabla 4. Alimentación de Phytotoma raimondii a lo largo de año en El Gramadal y otros lugares.

\begin{tabular}{|c|c|c|c|c|c|c|c|c|c|c|c|c|c|}
\hline Nombre científico * (común) & e & $\mathbf{f}$ & $\mathbf{m}$ & $\mathbf{a}$ & $\mathbf{m}$ & jn & $\mathrm{j} 1$ & $\mathbf{a}$ & $\mathbf{n}$ & d & por & Lugar & autor \\
\hline $\begin{array}{l}\text { Prosopis pallida } \\
\text { (algarrobo) }\end{array}$ & $\mathrm{f}, \mathrm{fr}$ & $\mathrm{f}, \mathrm{fr}$ & $\mathrm{f}, \mathrm{fr}$ & br & br & br & & & & & $\mathrm{H}$ & Talara & $\mathrm{CA}$ \\
\hline Prosopis juliflora & br & & & & & & & & & & $\mathrm{O}$ & Talara & CA \\
\hline Prosopis pallida & $\mathrm{f}$ & & & & br & br & br & br & & $\mathrm{f}$ & $\mathrm{O}$ & Huarmey & $\mathrm{MR}$ \\
\hline $\begin{array}{l}\text { Grabowskia boerhaviifolia } \\
\text { (palo negro) }\end{array}$ & fr & fr & $\mathrm{fr}, \mathrm{h}$ & $\mathrm{fr}, \mathrm{h}$ & $\mathrm{fr}$ & & & & & $\mathrm{fr}$ & $\mathrm{O}$ & Huarmey & MR \\
\hline Grabowskia boerhaviifolia & & & $\mathrm{f}, \mathrm{frbr}, \mathrm{h}$ & & & & $\mathrm{f}, \mathrm{frbr}, \mathrm{h}$ & & & & $\mathrm{O}$ & Virú & LP \\
\hline $\begin{array}{l}\text { Acacia huarango/ } \\
\text { macracantha (huarango) }\end{array}$ & br & & & & & & & & & & $\mathrm{O}$ & Talara & $\mathrm{CA}$ \\
\hline $\begin{array}{l}\text { Capparis crotonoides } \\
\text { (satuyo) }\end{array}$ & $\mathrm{fr}, \mathrm{s}$ & $\mathrm{s}$ & $\mathrm{s}$ & & & & & & & & $\mathrm{H}$ & Talara & $\mathrm{CA}$ \\
\hline $\begin{array}{l}\text { Capparis scabrida } \\
\text { (zapote) }\end{array}$ & & & & $\mathrm{f}$ & $\mathrm{f}$ & $\mathrm{f}$ & & & & & $\mathrm{H}$ & Talara & CA \\
\hline Capparis scabrida & & & & & & & & & $\mathrm{h} ?$ & & $\mathrm{O}$ & Huarmey & MR \\
\hline $\begin{array}{l}\text { Maytenus octogona } \\
\text { (realengo) }\end{array}$ & & & & $\mathrm{h}$ & $\mathrm{h}$ & $\mathrm{h}$ & & & & & $\mathrm{H}$ & Talara & CA \\
\hline $\begin{array}{l}\text { Capparis ovalifolia } \\
\text { (vichayo) }\end{array}$ & br & & & & & & & & & & $\mathrm{O}$ & Talara & $\mathrm{CA}$ \\
\hline $\begin{array}{l}\text { Cordia lutea } \\
\text { (overo) }\end{array}$ & $\mathrm{f}$ & $\mathrm{f}$ & $\mathrm{f}$ & & & & & & & & $\mathrm{O}$ & Talara & $\mathrm{CA}$ \\
\hline $\begin{array}{l}\text { Loxopterigium huasango } \\
\text { (hualtaco) }\end{array}$ & br & & & & & & & & & & $\mathrm{O}$ & Talara & $\mathrm{CA}$ \\
\hline $\begin{array}{l}\text { Psitacanthus chanduyensis } \\
\text { (suelda con suelda) }\end{array}$ & $\mathrm{f}$ & $\mathrm{f}$ & $\mathrm{f}$ & & & & & & & & $\mathrm{O}$ & Talara & $\mathrm{CA}$ \\
\hline
\end{tabular}

* Diaz 1995

$\mathrm{f}=\mathrm{flor}, \mathrm{fr}=$ fruto, $\mathrm{br}=$ brote de hoja, $\mathrm{h}=$ hojas, $\mathrm{s}=$ semillas. $\mathrm{H}=$ en heces, $\mathrm{O}=$ Observado comiendo. $\mathrm{CA}=\mathrm{C}$. Abramonte 2007, LP=L.Pollack 2009, MR= este trabajo.

\section{Discusión}

La información de la historia natural de la cortarrama peruana en aspectos básicos como su alimentación y reproducción está aumentando con datos de diferentes lugares de su distribución. En cuanto al hábitat hemos visto que para el caso de El Gramadal el ave vive en un matorral desértico aunque también se le ha reportado en los llamados bosques secos abiertos con sotobosque arbustivo y similar composición de plantas (Schulenberg et al. 2010).

Hemos observado que en El Gramadal el ave se alimenta de al menos dos especies vegetales, algarrobo y palo negro. De mayo a enero están disponibles los brotes y flores de algarrobo y el resto de los meses el palo negro tiene frutos maduros y abundantes, sobre todo de noviembre a junio. Es decir, durante todo el año al menos alguna de las dos especies sería la fuente principal de alimento. Además hay que considerar que ambas especies son abundantes o suficientes en el área.

Comparando con la lista de Talara de Abramonte (2007), quien también observó que se alimenta principalmente de algarrobo con una frecuencia del $73 \%$ (flores y frutos de enero a marzo y brotes de abril a junio; no se estudió de julio a diciembre), en El Gramadal, hay otras plantas presentes de las que podría alimentarse, como flores (pistilos) y brotes tiernos de zapote Capparis scabrida o brotes de faique Acacia sp. aunque nosotros no lo hemos observado. Abramonte (2007) también refiere que el ave se alimenta de las flores del overo Cordia lutea, pero esta planta sólo se encuentra en El Gramadal 2. Es decir en ausencia de brotes y flores de algarrobo o frutos de palo negro, el ave podría alimentarse de estas otras plantas, aunque algunas están muy dispersas y son escasas en El Gramadal.

En la Tabla 4, se presenta una lista de las especies de plantas de las que se conoce que se alimenta. Es interesante que la dieta de una de las tres especies congéneres, $P$. rutila, en Santa Fe, Argentina (De la Peña \& Pensiero 2003) incluye 9 especies y entre ellas también consume hojas (septiembre) y frutos (junio a octubre) de Prosopis alba y hojas (octubre a noviembre) de Grabowskia duplicata. Si lo comparamos con las estaciones en El Gramadal vemos que durante los meses de invierno P. raimondii también se alimenta de brotes de Prosopis pallida, mientras que en los meses de verano lo hemos visto alimentándose de frutos de Grabowskia boerhaaviifolia. Phytotoma rutila en Córdoba, Argentina (Bucher et al. 2003) consumía Prosopis alba y P. nigra a lo largo del ańo pero con menores valores en verano; de las 4 especies que más consumia (Lycium cestroides, Prosopis spp. Celtis tala y Schinus longifolius), Lycium (SOLANACEA) tenía los valores más altos de proteína a lo largo del año pero Prosopis spp. alcanzaba valores similares en el invierno y primavera. Para Prosopis, la hemicelulosa alcanzaba niveles más altos en el verano $(22 \%)$ que en el resto del ańo (1,8 a 13\%). Un análisis de regresión muestra que el contenido de fenoles $(\mathrm{r}=-0,64, \mathrm{P}=0,01)$ y proteínas $(\mathrm{r}=0,59, \mathrm{P}=0,03)$ eran los factores que más relación tenían con la variación en la composición de la dieta. Es lógico que para las aves folívoras haya selección por la mayor cantidad 
de proteína ya que las hojas en general tienen poco contenido de éstas; más aún, hay una relación negativa entre el contenido de fenoles y proteínas (Robbins 1981).

Sobre la importancia de El Gramadal para la reproducción, se ha visto que $P$. raimondii logra tener éxito aun cuando su hábitat sea de pequeñas dimensiones, siempre y cuando la composición y estructura vegetal de este no sea alterada y contenga los recursos mínimos necesarios. Esta consideración tiene importancia en proyectos de reforestación con algarrobo o para restauración ecológica, en los que también se debería considerar otras especies útiles para la cortarrama peruana como el palo negro. Además, información sobre la distancia entre plantas de importancia para el ave a lo largo del ańo es relevante en el momento de plantear el diseño de las parcelas a reforestar o restaurar.

El Gramadal contiene dos plantas que parecen cruciales para la presencia y reproducción de la cortarrama, el palo negro o canutillo que garantizaría no solo el alimento si no su éxito reproductivo y el algarrobo que aseguraría el alimento tanto a los adultos como a los individuos jóvenes durante la mayor parte del año. La presencia y abundancia de estas dos especies vegetales ha permitido que El Gramadal contenga la mayor densidad de cortarramas peruanas y sea uno de los mejores sitios para esta especie de los 16 lugares visitados donde la hemos encontrado a lo largo de la costa peruana (Romo \& Rosina en prep). Este estudio ilustra la importancia de medir los recursos útiles a una especie para entender su presencia y abundancia y en algunos casos su efecto en la territorialidad. En el caso de El Gramadal, con distancias entre nidos de menos de $100 \mathrm{~m}$ vemos que varias parejas podrían estar visitando o compartiendo los mismos algarrobos del lugar ya que se han encontrado 5 nidos relativamente cercanos al mismo tiempo en el 2011.

Nuestros resultados confirman la gran importancia de conservar El Gramadal ya que no solo es un buen hábitat sino también aquí se ha observado una buena reproducción de la especie por 3 años consecutivos. Este lugar es además el límite sur de la distribución del ave y uno de los pocos ejemplos de matorral desértico espinoso aun intacto en la costa peruana. De este tipo de hábitat, sólo quedan unos pocos parches, muy pequeños y muy alejados entre sí.

\section{Agradecimientos}

Agradecemos a Michael Tweddle por las estupendas fotografías que acompañan el artículo y que son un testimonio de la biología del ave. A nuestros asistentes Pablo César Romo y Lady Amaro. A Rob Williams quien nos informó sobre el lugar de El Gramadal. A Manuel Plenge quien con la amabilidad que lo caracteriza nos facilitó bibliografía y revisó nuestro manuscrito. A Angel Servat quien nos brindó alojamiento y grata hospitalidad en su casa. Mónica Romo expresa que esta investigación ha sido hecha fuera de sus actividades profesionales en la Agencia de Cooperación Internacional de los Estados Unidos -USAID.

\section{Literatura citada}

Abramonte C. 2007. Conducta reproductiva y dieta de Phytotoma raimondii (TACZANOWSKI, 1883) "Cortarrama peruana” en el bosque seco de Talara. Tesis para optar el título profesional de biólogo. Universidad Nacional de Piura.

Bucher E.A, D.Tamburini, A.Abril \& P.Torres. 2003. Folivory in the white-tipped plantcutter Phytotoma rutila: seasonal variations in diet composition and quality. Journal of Avian Biology 34: 211-216, 2003.

Clements J. \& N. Shany. 2001. A field guide to the birds of Peru. Ibis Publishing Co, Verona - Italy.

De la Peña, M \& J.Pensiero. 2003. Contribución de la flora en los hábitos alimentarios de las aves en un bosque del centro de la provincia de Santa Fe, Argentina. Ornitología Neotropical 14: 499-513, 2003.

Diaz, A. 1995. Los Algarrobos. Concytec. 207 pp.

Flanagan J.N.M. \& B. Millen 2008. First nest and eggs records of Peruvian Plantcutter, Phytotoma raimondii by O.D.Boggs Bull. B.O.C. 128(4):271.

Flanagan J.N.M, G. Engblom, I. Franke, T. Valqui \& F. Angulo 2009. Distribution of the Peruvian Plantcutter Phytotoma raimondii (Passeriformes: Cotingidae). Rev.per.biol. 16(2): 175-182.

Robbins, C. T. 1981. Estimation of the relative protein cost of reproduction in birds. Condor 83:177-179

Rosina M. \& M. Romo. 2010. Hallazgo de dos nidos activos de Phytotoma raimondii, Taczanowski, 1883, cortarrama peruana. Rev. Per. Biol 17(2): 257-259.

Schulenberg T.S, D.F. Stotz, D.E. Lane, J.P. O’Neill \& T.A. Parker III. 2010. Aves de Perú. Serie Biodiversidad Corbidi 01. Lima, Perú. 
